\title{
Seroprevalence and associated risk factors of Toxocara infection in Korean, Manchu, Mongol, and Han ethnic groups in northern China
}

\author{
G.-L. YANG ${ }^{1} \dagger$, X.-X. ZHANG ${ }^{1} \dagger$, C.-W. SHI ${ }^{1}$, W.-T. YANG ${ }^{1}$, Y.-L. JIANG ${ }^{1}$, \\ Z.-T. WEI ${ }^{3}$, C.-F. WANG ${ }^{1}$ AND Q. ZHAO ${ }^{2 *}$ \\ ${ }^{1}$ College of Animal Science and Technology, Jilin Provincial Engineering Research Center of Animal Probiotics, \\ Jilin Agricultural University, Changchun, Jilin Province, PR China \\ ${ }^{2}$ College of Animal Science and Technology, Chanchun Dci-Tech University, Shuangyang, Jilin Province, PR \\ China \\ ${ }^{3}$ The First Hospital, Jilin University, Changchun Jilin Province, PR China
}

Received 2 March 2016; Final revision 30 June 2016; Accepted 30 June 2016; first published online 26 July 2016

\section{SUMMARY}

Toxocariasis is a very prevalent zoonotic disease worldwide. Recently, investigators have focused more on Toxocara spp. seroprevalence in humans. Information regarding Toxocara seroprevalence in people from different ethnic backgrounds in China is limited. For this study, blood samples were collected from a total of 802 Han, 520 Korean, 303 Manchu, and 217

Mongol subjects from Jilin and Shandong provinces. The overall Toxocara seroprevalence was $16 \cdot 07 \%$ (14.21\% Han, 20.58\% Korean, $11 \cdot 22 \%$ Manchu, 18.89\% Mongol). Living in suburban or rural areas, having dogs at home, exposure to soil, and consumption of raw/undercooked meat were risk factors for Toxocara infection. Exposure to soil was identified as the major risk factor for Toxocara seropositivity in all of the tested ethnicities. To the best of our knowledge, this is the first report concerning Toxocara infection in Manchus and Mongols in China. The present study provided baseline data for effective prevention strategies of toxocariasis in northeast China and recommends improvements in personal hygiene standards to achieve this goal.

Key words: China, Korean ethnicity, Manchu ethnicity, Mongol ethnicity, seroepidemiology, Toxocara.

\section{INTRODUCTION}

Toxocariasis, a highly underrated and neglected disease, is caused by Toxocara canis and Toxocara cati, which are important intestinal nematodes of dogs and cats, respectively [1-4]. Of these, T. canis is the most common causative agent of human toxocariasis [5-8]. Humans acquire toxocariasis by accidental ingestion of embryonated T. canis eggs (from water or

\footnotetext{
* Author for correspondence: Professor Q. Zhao, College of Animal Science and Technology, Changchun Dci-Tech University, 1699 Donghua Street, Shuangyang 130600, China. (Email: zhaoquan0825@163.com)

$\dagger$ These authors contributed equally to this work.
}

soil contaminated by infected cat/dog faeces) or encapsulated larvae (from raw/undercooked meat); toxocariasis usually does not show any symptoms [3, $9,10]$. However, there are four general manifestations of toxocariasis: covert, visceral larva migrans, ocular larva migrans and neurotoxocariosis. Once the infective $T$. canis larvae are ingested, the helminths migrate into internal organs, which may lead to severe illnesses including neurotoxocarosis, visceral larva migrans, and eosinophilic meningoencephalitis $[1,2,5,6,11-14]$.

Toxocariasis is of increasing concern because of the increasing numbers of pet and stray dogs. Indeed, with the improvement in the standard of living, increasing numbers of people are acquiring pet dogs, which are 
potentially important sources of $T$. canis infections $[10,15,16]$. In addition to the high resistance of T. canis eggs to harsh environmental conditions, pet dogs have increased the risk of this infection in humans. Consequently, investigators have focused more on the global seroprevalence of Toxocara spp. in humans [10, 13, 17-22]. There are 56 ethnicities in China [23]. However, information concerning T. canis seroprevalence in different ethnic groups is limited. To our knowledge, Toxocara infection has been reported in several groups: children in Sichuan (11.49\%) [24], Shandong, and Jilin provinces (19.3\%) [3]; clinically healthy individuals, pregnant women and psychiatric patients in Shandong Province (12.25\%) [6]; and asthma patients in Nanjing City [25], but these studies were only related to the Han and Korean ethnic groups.

Because of the possible impact of different cultural backgrounds, customs, and habits, investigation of the risk factors of seroprevalence of $T$. canis infection in different ethnic groups is essential. Most Koreans in China live in Yanbian Korean Autonomous Prefecture, Jilin Province. Dog meat is a popular food for Koreans and is usually eaten either well cooked or raw. Inner Mongolia and northeast China are the most common residences of Mongols. Although Mongols have adapted to modern society, the characteristics that drive people to migrate to where there is water and grass are more or less maintained in this ethnic group. The Han ethnic group is the largest population group in China. Some Hans live together with other ethnic groups and have adopted similar living and dietary habits. For example, in rural areas of northeast China, some Hans also like to eat raw/uncooked meat and drink untreated well or river water. Manchus are now living across the country with similar diets and culture to the Hans. The present study aims to estimate the $T$. canis seroprevalence in four main ethnic groups (Han, Manchu, Mongol, Korean) in Jilin and Shandong provinces (northeastern and eastern China) and assess the risk factors associated with infection in these ethnic groups. This study will provide useful baseline information for planning effective prevention and control of $T$. canis infection in different ethnic groups in China.

\section{MATERIALS AND METHODS}

\section{Sample collection and laboratory tests}

This study was approved by the Ethics Committee of Jilin Agricultural University (Approval No. JAUAEC2013-003). A total of 1842 blood samples were collected from Han $(n=802)$, Korean $(n=520)$,
Manchu ( $n=303)$, and Mongol $(n=217)$ subjects in Jilin and Shandong urban and rural areas by medically trained staff between June 2013 and August 2015. The purpose and procedures of the study were explained to all participants, and written informed consent was obtained from each of them. Volunteers/guardians provided informed consent on behalf of all child participants. The sera were collected with agreement from the volunteers. Blood samples $(5 \mathrm{ml})$ were taken from the elbow vein of each participant using a plain tube. Serum samples were separated by centrifuging at $4000 \mathrm{rpm}$ for $8 \mathrm{~min}$. The sera were collected in Eppendorf tubes and stored at $4{ }^{\circ} \mathrm{C}$ for $24-72 \mathrm{~h}$ until transportation in an ice box to the Laboratory of Veterinary Parasitology, College of Animal Science and Technology, Jilin Agricultural University, Changchun, Jilin Province, where they were kept at $-20^{\circ} \mathrm{C}$ until analysed.

\section{Serological tests}

All the serum samples were tested for Toxocara IgG antibodies using a commercially available enzyme immunoassay Toxocara kit (Diagnostic Automation Inc., USA). The positive and negative controls were supplied in the kit and used in each test. When the absorbance reading was $\geqslant 0 \cdot 3$ OD units, the sample was considered to be positive. Doubtful samples were retested. All the operations were performed according to the manufacturer's instructions [6, 13].

\section{Statistical analysis}

The variation in seroprevalence of Toxocara-infected participants $(y)$ of different variables including age $(x 1)$, gender $(x 2)$, residential place $(x 3)$, residential area $(x 4)$, ethnicity $(x 5)$, cat at home $(x 6), \operatorname{dog}$ at home $(x 7)$, water sources $(x 8)$, consumption of raw/undercooked meat $(x 9)$, and exposure to soil $(x 10)$ were analysed by $\chi^{2}$ test using SAS version 9.1 (SAS Institute Inc., USA). Each variable was included in a binary logic model as an independent variable by multivariate regression analysis. $P<$ 0.05 was considered statistically significant. Odds ratios (ORs) and their $95 \%$ confidence intervals (CIs) were estimated to examine the strength of the association between Toxocara positivity and the selected condition.

\section{RESULTS}

\section{Seroprevalence in different ethnic groups}

The overall seroprevalence of Toxocara in the present study was $16 \cdot 07 \%$. The seroprevalence ranged from a 
minimum of $11 \cdot 22 \%$ in Manchus to a maximum of $20.58 \%$ in Koreans (Table 1). In the Han ethnic group, the seroprevalences of Toxocara in Weihai, Qingdao, and Changchun cities were $14.32 \%$, $13 \cdot 81 \%$, and $14 \cdot 50 \%$, respectively (Table 1 ). The highest $(18.75 \%)$ seroprevalence of Toxocara infection was found in the 30-39 years age group (Table 1). Females have a higher seroprevalence $(16 \cdot 67 \%)$ than males $(11 \cdot 35 \%$; Table 1). In the Korean ethnic group, Toxocara seroprevalences were $24 \cdot 42 \%, 15 \cdot 26 \%$, and $22.12 \%$ in Weihai, Qingdao, and Changchun cities, respectively (Table 1). Koreans aged 50-59 years had the highest $(24 \cdot 05 \%)$ seroprevalence (Table 1). In the Manchu ethnic group, Toxocara seroprevalences were $16.67 \%, 14.61 \%$, and $11.49 \%$ in Weihai, Qingdao, and Changchun cities, respectively (Table 1). T. canis seroprevalences in Manchus living in urban and suburban/rural areas were $8 \cdot 24 \%$ and $20 \cdot 30 \%$, respectively (Table 1). The seroprevalences in different age groups varied from $5 \cdot 41 \%$ to $23 \cdot 81 \%$ (Table 1). In the Mongol ethnic group, Toxocara seroprevalences in Weihai, Qingdao, and Changchun were 18.06\%, $20 \cdot 69 \%$ and $12 \cdot 93 \%$, respectively (Table 1 ). Toxocara seroprevalence in different age groups varied from $7 \cdot 41 \%$ to $25 \cdot 00 \%$ (Table 1 ).

\section{Risk factor analysis}

For the study populations, the effects of age, gender, city, residential area, ethnicity, cat at home, dog at home, water sources, consumption of raw/undercooked meat, and exposure to soil contaminated with Toxocara eggs based on seropositivity were considered in the evaluation by the forward stepwise logistic regression analysis using Fisher's scoring technique; this was described by the equation

$$
\begin{aligned}
y= & 0.5049 \times 4+0.4729 \times 7+0.3555 \times 9 \\
& +1.2599 \times 10-2.2364 .
\end{aligned}
$$

Four variables, including living in suburban or rural areas, dog at home, exposure to soil, and consumption of raw/undercooked meat were found to be potential risk factors for Toxocara infection, for which the ORs were 1.657 (95\% CI 1.209-2.272), 1.605 (95\% CI $1 \cdot 192-2 \cdot 161), 1 \cdot 427(95 \%$ CI $1 \cdot 106-1 \cdot 840)$, and $3 \cdot 525$ (95\% CI $2 \cdot 523-4 \cdot 925)$, respectively.

For the Han group, logistic regression analysis showed that gender (OR $1 \cdot 56,95 \%$ CI $1 \cdot 04-2 \cdot 35$, $P=0 \cdot 0306$ ), dog at home (OR $1 \cdot 73,95 \%$ CI $1 \cdot 11-$ $2 \cdot 71, P=0 \cdot 0151$ ), and exposure to soil (OR 2.96, $95 \%$ CI $1.92-4.58, \quad P<0.0001)$ were significantly associated with Toxocara infection (Table 2). Meanwhile, exposure to soil (OR 1.68, 95\% CI $1 \cdot 09-2 \cdot 60, \quad P=0.0187)$ and consumption of raw $/$ undercooked meat (OR 1·78, 95\% CI 1·16-2.74, $P=$ $0 \cdot 0080)$ were identified as being associated with Toxocara infection in Koreans (Table 2). Furthermore, residential area (OR 2.84, 95\% CI $1 \cdot 42-5 \cdot 57, P=0 \cdot 0023$ ), water sources (OR $2 \cdot 94,95 \%$ CI $1 \cdot 49-5 \cdot 78, P=0 \cdot 0013$ ), and exposure to soil (OR $=3 \cdot 67,95 \%$ CI $1 \cdot 68-7 \cdot 98, P=0 \cdot 0006)$ were identified as the major risk factors for Toxocara seropositivity in Manchus (Table 2). In the present study, exposure to soil (OR 2·87, 95\% CI 1·27-6.49, $P=0 \cdot 0090$ ) was the only factor associated with Toxocara seropositivity in Mongols (Table 2).

\section{DISCUSSION}

The overall $T$. canis seroprevalence in the study populations was $16 \cdot 07 \%(95 \%$ CI $14 \cdot 39-17 \cdot 75)$. This rate is higher than the $11.49 \%$ reported in children in Chengdu, China [24], $12 \cdot 25 \%$ in clinically healthy individuals, pregnant women, psychiatric patients in Shandong Province, Eastern China [6], 6.4\% in pregnant women in southern Brazil [10], 12.03\% in the Roma and non-Roma populations of Eastern Slovakia [22], 15.5\% in Brazilian schoolchildren [21], and $4.7 \%$ in psychiatric patients in Mexico [13]. However, the rate is lower than the $19 \cdot 3 \%$ reported in children in Shandong and Jilin provinces [3], $48.4 \%$ in a large urban setting in northeast Brazil [19], 50.6\% in southern Brazil [20], 40.6\% in mountain aboriginal adults in Taiwan [17], and $86.75 \%$ among primary schoolchildren in the capital area of the republic of the Marshall Islands [18]. Many factors, including diagnostic methods, geographical conditions, the timing of sample collection, sample sizes, sanitation, and life style of the evaluated population, might have contributed to the differences observed in seroprevalence rates.

In the present study, Koreans showed the highest T. canis seroprevalence of the four ethnic groups studied, but the differences in the rates were not statistically significant in different ethnic groups $(P>0 \cdot 05)$. However, this result is in contrast with the findings of a previous study in which Koreans were reported to have lower seroprevalence than Hans [6]; this difference could be attributed to the different sample sizes, different sampling times, and individual ethnic differences. 
Table 1. Socio-demographic and risk factors associated with Toxocara seropositivity in different ethnic groups

\begin{tabular}{|c|c|c|c|c|c|c|c|c|c|c|c|c|c|c|c|c|}
\hline \multirow[b]{2}{*}{ Variable } & \multicolumn{4}{|c|}{ Han ethnicity } & \multicolumn{4}{|c|}{ Korean ethnicity } & \multicolumn{4}{|c|}{ Manchu ethnicity } & \multicolumn{4}{|c|}{ Mongol ethnicity } \\
\hline & $\begin{array}{l}\text { No. } \\
\text { tested }\end{array}$ & $\begin{array}{l}\text { No. } \\
\text { positive }\end{array}$ & $\%$ & $P$ value & $\begin{array}{l}\text { No. } \\
\text { tested }\end{array}$ & $\begin{array}{l}\text { No. } \\
\text { positive }\end{array}$ & $\%$ & $P$ value & $\begin{array}{l}\text { No. } \\
\text { tested }\end{array}$ & $\begin{array}{l}\text { No. } \\
\text { positive }\end{array}$ & $\%$ & $P$ value & $\begin{array}{l}\text { No. } \\
\text { tested }\end{array}$ & $\begin{array}{l}\text { No. } \\
\text { positive }\end{array}$ & $\%$ & $P$ value \\
\hline \multicolumn{17}{|l|}{ Age group (years) } \\
\hline$\leqslant 19$ & 90 & 13 & $14 \cdot 44$ & \multirow[t]{6}{*}{$0 \cdot 7074$} & 58 & 11 & $18 \cdot 97$ & \multirow[t]{6}{*}{$0 \cdot 9204$} & 53 & 7 & $13 \cdot 21$ & \multirow[t]{6}{*}{$0 \cdot 3025$} & 27 & 2 & $7 \cdot 41$ & \multirow[t]{6}{*}{$0 \cdot 1707$} \\
\hline $20-29$ & 61 & 10 & $16 \cdot 39$ & & 55 & 12 & $21 \cdot 82$ & & 27 & 3 & $11 \cdot 11$ & & 28 & 4 & $14 \cdot 29$ & \\
\hline $30-39$ & 112 & 21 & $18 \cdot 75$ & & 63 & 13 & $20 \cdot 64$ & & 37 & 2 & $5 \cdot 41$ & & 30 & 7 & $23 \cdot 33$ & \\
\hline $40-49$ & 219 & 30 & $13 \cdot 70$ & & 145 & 26 & $17 \cdot 93$ & & 90 & 12 & $13 \cdot 33$ & & 59 & 5 & $8 \cdot 48$ & \\
\hline $50-59$ & 137 & 17 & $12 \cdot 41$ & & 79 & 19 & $24 \cdot 05$ & & 54 & 7 & $12 \cdot 96$ & & 36 & 9 & $25 \cdot 00$ & \\
\hline$\geqslant 60$ & 183 & 23 & $12 \cdot 57$ & & 120 & 26 & $21 \cdot 67$ & & 42 & 10 & $23 \cdot 81$ & & 37 & 7 & $18 \cdot 92$ & \\
\hline \multicolumn{17}{|l|}{ Gender } \\
\hline Male & 370 & 42 & $11 \cdot 35$ & \multirow[t]{2}{*}{$0 \cdot 0316$} & 224 & 51 & $22 \cdot 77$ & \multirow[t]{2}{*}{$0 \cdot 2823$} & 137 & 21 & $15 \cdot 33$ & \multirow[t]{2}{*}{$0 \cdot 4061$} & 95 & 18 & $18 \cdot 95$ & \multirow[t]{2}{*}{$0 \cdot 2409$} \\
\hline Female & 432 & 72 & $16 \cdot 67$ & & 296 & 56 & $18 \cdot 92$ & & 166 & 20 & $12 \cdot 05$ & & 122 & 16 & $13 \cdot 12$ & \\
\hline \multicolumn{17}{|l|}{ Location } \\
\hline Changchun & 131 & 19 & $14 \cdot 50$ & \multirow{3}{*}{0.9796} & 113 & 25 & $22 \cdot 12$ & \multirow{3}{*}{$0 \cdot 0668$} & 148 & 17 & $11 \cdot 49$ & \multirow{3}{*}{$0 \cdot 5567$} & 116 & 15 & $12 \cdot 93$ & \multirow{3}{*}{0.4673} \\
\hline Qingdao & 210 & 29 & $13 \cdot 81$ & & 190 & 29 & $15 \cdot 26$ & & 89 & 13 & $14 \cdot 61$ & & 29 & 6 & $20 \cdot 69$ & \\
\hline Weihai & 461 & 66 & $14 \cdot 32$ & & 217 & 53 & $24 \cdot 42$ & & 66 & 11 & $16 \cdot 67$ & & 72 & 13 & $18 \cdot 06$ & \\
\hline \multicolumn{17}{|l|}{ Residential area } \\
\hline Urban & 487 & 64 & $13 \cdot 14$ & \multirow{2}{*}{$0 \cdot 2794$} & 300 & 59 & $19 \cdot 67$ & \multirow{2}{*}{$0 \cdot 5488$} & 170 & 14 & $8 \cdot 24$ & $0 \cdot 0023$ & 123 & 20 & $16 \cdot 26$ & $0 \cdot 7838$ \\
\hline Suburban or rural & 315 & 50 & $15 \cdot 87$ & & 220 & 48 & $21 \cdot 81$ & & 133 & 27 & $20 \cdot 30$ & & 94 & 14 & $14 \cdot 89$ & \\
\hline Cat at home & & & & & & & & & & & & & & & & \\
\hline Yes & 174 & 24 & $13 \cdot 79$ & $0 \cdot 8573$ & 122 & 24 & $19 \cdot 67$ & $0 \cdot 7775$ & 59 & 12 & $20 \cdot 34$ & 0.0885 & 47 & 8 & $17 \cdot 02$ & $0 \cdot 7731$ \\
\hline No & 628 & 90 & $14 \cdot 33$ & & 398 & 83 & $20 \cdot 85$ & & 244 & 29 & $11 \cdot 89$ & & 170 & 26 & $15 \cdot 29$ & \\
\hline Dog at home & & & & & & & & & & & & & & & & \\
\hline Yes & 164 & 33 & $20 \cdot 12$ & $0 \cdot 0151$ & 99 & 23 & $23 \cdot 23$ & $0 \cdot 5019$ & 69 & 12 & $17 \cdot 39$ & $0 \cdot 2861$ & 33 & 8 & $24 \cdot 24$ & $0 \cdot 1411$ \\
\hline No & 638 & 81 & $12 \cdot 70$ & & 421 & 85 & $26 \cdot 48$ & & 234 & 29 & $12 \cdot 39$ & & 184 & 26 & $14 \cdot 13$ & \\
\hline $\begin{array}{l}\text { Consumption of raw/ } \\
\text { undercooked meat }\end{array}$ & & & & & & & & & & & & & & & & \\
\hline Yes & 330 & 49 & $14 \cdot 85$ & $0 \cdot 6672$ & 242 & 62 & $25 \cdot 62$ & $0 \cdot 0080$ & 136 & 22 & $16 \cdot 18$ & $0 \cdot 2245$ & 87 & 16 & $18 \cdot 39$ & $0 \cdot 3667$ \\
\hline No & 472 & 65 & $13 \cdot 77$ & & 278 & 45 & $16 \cdot 19$ & & 167 & 19 & $11 \cdot 38$ & & 130 & 18 & $13 \cdot 85$ & \\
\hline Exposure to soil & & & & & & & & & & & & & & & & \\
\hline Yes & 401 & 82 & $20 \cdot 45$ & $<0 \cdot 0001$ & 273 & 67 & $24 \cdot 54$ & $0 \cdot 0187$ & 161 & 32 & $19 \cdot 88$ & $0 \cdot 0006$ & 115 & 25 & $21 \cdot 74$ & $0 \cdot 0090$ \\
\hline No & 401 & 32 & $7 \cdot 98$ & & 247 & 40 & $16 \cdot 19$ & & 142 & 9 & $6 \cdot 34$ & & 102 & 9 & $8 \cdot 82$ & \\
\hline Source of drinking wat & & & & & & & & & & & & & & & & \\
\hline Tap & 536 & 76 & $14 \cdot 18$ & 0.9675 & 352 & 68 & $19 \cdot 32$ & $0 \cdot 3041$ & 187 & 16 & $8 \cdot 56$ & $0 \cdot 0013$ & 156 & 29 & $18 \cdot 59$ & $0 \cdot 0583$ \\
\hline Well+river & 266 & 38 & $14 \cdot 29$ & & 168 & 39 & $23 \cdot 21$ & & 116 & 25 & $21 \cdot 55$ & & 61 & 5 & $8 \cdot 20$ & \\
\hline Total & 802 & 114 & $14 \cdot 21$ & & 520 & 107 & $20 \cdot 58$ & & 303 & 41 & $11 \cdot 22$ & & 217 & 34 & $18 \cdot 89$ & \\
\hline
\end{tabular}


Table 2. Odds ratio of the risk factors associated with seropositivity to Toxocara in different ethnic groups in northern China

\begin{tabular}{|c|c|c|c|c|}
\hline Ethnicity & Variables & OR & $95 \% \mathrm{CI}$ & $P$ value \\
\hline \multirow[t]{9}{*}{ Han } & Gender & & & \\
\hline & Male & Reference & & \\
\hline & Female & 1.56 & $1 \cdot 04-2 \cdot 35$ & $0 \cdot 0316$ \\
\hline & Dog at home & & & \\
\hline & No & Reference & & \\
\hline & Yes & $1 \cdot 73$ & $1 \cdot 11-2 \cdot 71$ & $0 \cdot 0151$ \\
\hline & Exposure to soil & & & \\
\hline & No & Reference & & \\
\hline & Yes & $2 \cdot 96$ & $1.92-4.58$ & $<0 \cdot 0001$ \\
\hline \multirow[t]{6}{*}{ Korean } & Exposure to soil & & & \\
\hline & No & Reference & & \\
\hline & Yes & 1.68 & $1 \cdot 09-2 \cdot 60$ & $0 \cdot 0187$ \\
\hline & $\begin{array}{l}\text { Consumption of raw/ } \\
\text { undercooked meat }\end{array}$ & & & \\
\hline & No & Reference & & \\
\hline & Yes & 1.78 & $1 \cdot 16-2 \cdot 74$ & $0 \cdot 0080$ \\
\hline \multirow[t]{9}{*}{ Manchu } & Residential area & & & \\
\hline & Urban & Reference & & \\
\hline & Suburban or rural & $2 \cdot 84$ & $1 \cdot 42-5 \cdot 57$ & $0 \cdot 0023$ \\
\hline & Water sources & & & \\
\hline & Tap & Reference & & \\
\hline & Well + river & $2 \cdot 94$ & $1 \cdot 49-5 \cdot 78$ & $0 \cdot 0013$ \\
\hline & Exposure to soil & & & \\
\hline & No & Reference & & \\
\hline & Yes & $3 \cdot 67$ & $1.68-7.98$ & $0 \cdot 0006$ \\
\hline \multirow{3}{*}{ Mongol } & Exposure to soil & & & \\
\hline & No & Reference & & \\
\hline & Yes & $2 \cdot 87$ & $1.27-6.49$ & $0 \cdot 0090$ \\
\hline
\end{tabular}

OR, Odds ratio; CI, confidence interval.

It is well known that dogs are the most important definitive host of $T$. canis. The parasite's eggs can survive not only in the faeces and fur of domestic dogs but also in soil and water after their faeces have been discharged into these areas $[6,21,26]$. Hence, dogs at home and exposure to contaminated soil were undoubtedly risk factors for $T$. canis infection in humans $[3,6]$. The present study has shown that exposure to soil is associated with $T$. canis infection in all four ethnic groups, which suggests that the problem of polluted soil is widespread in these areas.

However, only in the Han ethnic group were having dogs at home (OR 1.73, 95\% CI 1·11-2.71, $P=0 \cdot 0151$ ) associated with $T$. canis infection (Table 2). Actually, dogs at home and contact with dogs are not necessarily risk factors unless there is lack of essential measures (e.g. not washing hands) after having contact with dogs' faeces. The high $T$. canis burden in the Manchu ethnic group would be partially explained by this phenomenon. Manchus passionately raise dogs, especially in suburban and rural areas, where the majority of the people use untreated water from wells or rivers for drinking. The seroprevalence of T. canis was rather high in Manchus for those raising dogs and those living in rural areas. Furthermore, Han women handle raw meat and vegetables more frequently and spend more time with their pets than men, which may account for their significantly higher $T$. canis seroprevalence than the rate observed in males $(P=0 \cdot 0316)$. In China, the increasing number of pet dogs and inadequate inspection and quarantine measures enhance the potential toxocariasis risk for humans. In addition, the old Korean tradition of consuming dog meat and the habit of consuming raw/ undercooked meat, which may contain encapsulated larvae, across the groups can also be associated with the Toxocara infection in humans [6, 21, 27-29]. Improved and integrated strategies and measures are required for the effective prevention and control of toxocariasis in these ethnic groups in northern China. 


\section{CONCLUSION}

The present study has shown that the overall seroprevalence of Toxocara infection in the various ethnic groups examined from Jilin and Shandong provinces, northern China, was $16 \cdot 07 \%$. The lowest seropositivity $(11.22 \%)$ was recorded in the Manchu group, while the highest seropositivity (20.58\%) was recorded in Koreans. The study revealed the seroprevalence of Toxocara infection in Manchus and Mongols for the first time. Living in suburban or rural areas, dogs at home, exposure to soil, and consumption of raw/ undercooked meat are risk factors of Toxocara infection. Moreover, more attention should be given to the Han women and Manchus who come in contact with unboiled water. Hence, establishment of good health habits including washing hands before meals and after contact with soil, regular deworming of dogs, and eating well-cooked meat should be promoted. Investigation of soil contamination between cities should be further studied.

\section{ACKNOWLEDGEMENTS}

This work was supported by the National high-tech R\&D Programme of China (863 programme) (2013AA102806), National Natural Science Foundation of China (31272552, 31272541), Science and Technology Development Programme of Jilin Province (20160519011JH), Special Funds for Industrial Innovation of Jilin Province (2016C063) and the Key Scientific and Technological Project of Jilin Province (20140204068NY).

\section{DECLARATION OF INTEREST}

None.

\section{REFERENCES}

1. Chen $\mathbf{J}$, et al. Advances in molecular identification, taxonomy, genetic variation and diagnosis of Toxocara spp. Infection, Genetics and Evolution 2012; 12: 1344 1348.

2. Chen $\mathbf{J}$, et al. Canine and feline parasitic zoonoses in China. Parasites \& Vectors 2102; 5: 152.

3. Cong W, et al. Seroprevalence and risk factors of Toxocara infection among children in Shandong and Jilin provinces, China. Acta Tropica 2015; 152: 215-219.

4. Nijsse R, et al. Toxocara canis in household dogs: prevalence, risk factors and owners' attitude towards deworming. Parasitology Research 2015; 114: 561-569.
5. Manini MP, et al. Association between contamination of public squares and seropositivity for Toxocara spp. in children. Veterinary Parasitology 2012; 188: 48-52.

6. Cong W, et al. Toxocara seroprevalence among clinically healthy individuals, pregnant women and psychiatric patients and associated risk factors in Shandong Province, Eastern China. PLoS Neglected Tropical Diseases 2014; 8: e3082.

7. Gyang PV, et al. Seroprevalence, disease awareness, and risk factors for Toxocara canis infection among primary schoolchildren in Makoko, an urban slum community in Nigeria. Acta Tropica 2015; 146: 135-140.

8. Martínez M, et al. Seroprevalence and risk factors of toxocariasis in preschool children in Aragua state, Venezuela. Transactions of the Royal Society of Tropical Medicine and Hygiene 2015; 109: 579-88.

9. Campos-da-Silva DR, et al. Natural infection of freerange chickens with the ascarid nematode Toxocara sp. Parasitology Research 2015; 114: 4289-4293.

10. Santos PC, et al. The seropositivity of Toxocara spp. antibodies in pregnant women attented at the university hospital in southern Brazil and the factors associated with infection. PLOS ONE 2015; 10: e0131058.

11. Helsen G, Vandecasteele SJ, Vanopdenbosch LJ. Toxocariasis presenting as encephalomyelitis. Case Reports in Medicine 2011; 2011: 503913.

12. Demirci M, et al. Eosinophilic pneumonia due to toxocariasis: an adult case report. Türkiye Parazitolojii Dergisi 2012; 36: 258-259.

13. Alvarado-Esquivel C. Toxocara infection in psychiatric inpatients: a case control seroprevalence study. PLoS One 2013; 8: e62606.

14. Fan CK, Liao CW, Cheng YC. Factors affecting disease manifestation of toxocarosis in humans: genetics and environment. Veterinary Parasitology 2013; 193: 342-352.

15. Khademvatan $\mathbf{S}$, et al. PCR-based molecular characterization of Toxocara spp. using feces of stray cats: a study from Southwest Iran. PLoS ONE 2013; 8: e65293.

16. Kroten A, et al. Environmental contamination with Toxocara eggs and seroprevalence of toxocariasis in children of northeastern Poland. Parasitology Research 2016; 115:205-209.

17. Fan CK, et al. Seroepidemiology of Toxocara canis infection among mountain aboriginal adults in Taiwan. The American journal of Tropical Medicine and Hygiene 2004; 71: 216-221.

18. Fu CJ, et al. Seroepidemiology of Toxocara canis infection among primary schoolchildren in the capital area of the Republic of the Marshall Islands. BMC Infectious Diseases 2014; 14: 261.

19. Mendonça LR, et al. Seroprevalence and risk factors for Toxocara infection in children from an urban large setting in Northeast Brazil. Acta Tropica 2013; 128: 90-95.

20. Schoenardie ER, et al. Seroprevalence of Toxocara infection in children from southern Brazil. The Journal of Parasitology 2013; 99: 537-539.

21. Cassenote AJ, et al. Seroprevalence and modifiable risk factors for Toxocara spp. in Brazilian 
schoolchildren. PLoS Neglected Tropical Diseases 2014; 8: e2830.

22. Antolová D, et al. Seroprevalence of human Toxocara infections in the Roma and non-Roma populations of Eastern Slovakia: a cross-sectional study. Epidemiology and Infection 2015; 143: 2249-2258.

23. Zhang XX, et al. Seroprevalence and associated risk factors of Toxoplasma gondii infection in the Korean, Manchu, Mongol and Han ethnic groups in eastern and northeastern China. Epidemiology and Infection 2016; 144: 2018-2024.

24. Luo ZJ, et al. Detection of circulating antigens and antibodies in Toxocara canis infection among children in Chengdu, China. The Journal of Parasitology 1999; 85: $252-256$.
25. Li L, et al. Asthma and toxocariasis. Annals of Allergy, Asthma \& Immunology 2014; 113: 187-192.

26. Paoletti B, et al. Zoonotic parasites in feces and fur of stray and private dogs from Italy. Parasitology Research 2015; 114: 2135-2141.

27. Taira K, et al. Zoonotic risk of Toxocara canis infection through consumption of pig or poultry viscera. Veterinary Parasitology 2004; 121: 115-124.

28. Choi D, et al. Transmission of Toxocara canis via ingestion of raw cow liver: a cross-sectional study in healthy adults. Korean Journal of Parasitology 2012; 50: $23-27$.

29. Noh Y, et al. Meningitis by Toxocara canis after ingestion of raw ostrich liver. Journal of Korean Medical Science 2012; 27: 1105-1108. 